\title{
A SURVEY OF OH IN THE GALACTIC CENTRE REGION
}

\author{
P.J. BOYCE \\ Department of Physics and Astronomy, \\ University of Wales College of Cardiff \\ 5 The Parade, PO Box 913, Cardiff CF2 3YB
}

AND

R. J. COHEN

University of Manchester, Nuffield Radio Astronomy Laboratories, Jodrell Bank, Macclesfield, Cheshire SK11 9DL

\section{Introduction}

The galactic centre contains the largest concentration of molecular clouds in the Galaxy. The clouds in the central region are unusual in having large linewidths and masses, and large non-circular motions. Previous surveys of their distribution in the central region have been carried out in OH (Robinson \& McGee 1970; Cohen \& Few 1976), $\mathrm{H}_{2} \mathrm{CO}$ (Whiteoak \& Gardner 1979; Cohen \& Few 1981), CO (Bania 1977; Dame et al. 1987; Bally et al. 1987, 1988 ) and CS (Bally et al. 1987, 1988). The OH groundstate lines at $18 \mathrm{~cm}$ wavelength have certain advantages for such a survey. The $\mathrm{OH}$ lines appear in absorption against the galactic centre continuum sources, and against the continuum emission from the disk of the Galaxy. The absorption spectra are sensitive to relatively small molecular column densities. In addition they can give information on the relative positions of the molecular gas and the radio continuum sources. This paper describes results from an absorption line survey of the galactic centre region in the $\mathrm{OH}$ main lines at 1667.359 MHz and 1665.402 MHz (Boyce \& Cohen 1994). 


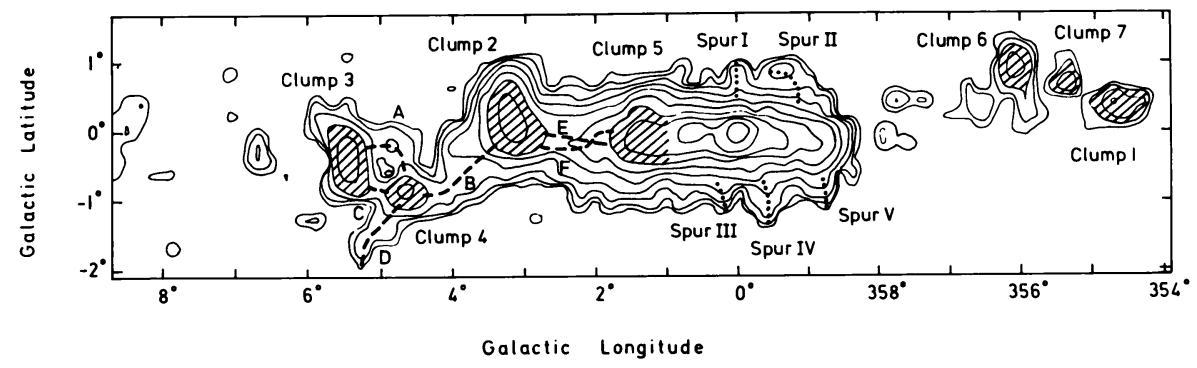

Figure 1. Map of integrated $\mathrm{OH}$ absorption with the positions of the broadline clumps (cross-hatched), filaments (dashed lines) and molecular spurs (solid lines) marked.

\section{Observations}

The survey was made using the 250ft Lovell Telescope at Jodrell Bank which has a beamwidth of 10 arcmin at $1666 \mathrm{MHz}$. Spectra were taken on a grid with 0.2 spacing. The entire region between $354^{\circ} \leq l \leq 8 . .^{\circ} 6$ and $-1 . \circ 0 \leq b \leq+1 . \circ 0$ was surveyed with extensions out to $b=-2 .{ }^{\circ} 0$ and $b=+1 . \circ 6$ at some longitudes. The spectra were taken using an autocorrelation spectrometer with spectral bandwidth $5 \mathrm{MHz}$, a velocity range of $900 \mathrm{~km} \mathrm{~s}^{-1}$ and a resolution of $3.1 \mathrm{~km} \mathrm{~s}^{-1}$. The rms noise level achieved was typically 80-90 mK. The data are presented by Boyce \& Cohen (1994) as a series of longitude-velocity $(l-V)$, latitude-velocity $(b-V)$ and longitude-latitude $(l-b)$ contour maps of line temperature and apparent opacity.

\section{Results}

The results are summarized here in Figures 1 and 2. Figure 1 shows the integrated $\mathrm{OH}$ absorption, with some the individual features labeled. Figure 2 shows a longitude-velocity map of the $\mathrm{OH}$ absorption integrated over all latitudes of the survey.

The $\mathrm{OH}$ survey clearly reveals the full extent of the molecular gas distribution in the galactic centre region. The molecular nuclear disk can be traced over the entire region $354^{\circ}<l<6^{\circ}$, corresponding to a projected diameter of $\sim 1.8 \mathrm{kpc}$. The structure is warped, lying preferentially below the galactic plane at positive longitudes, and above the plane at negative longitudes. The warp extends to at least $\pm 1 .{ }^{\circ} 6$ in latitude, corresponding to $\mathrm{z}$-distances of $\pm 240 \mathrm{pc}$. The warp is in the same sense as the warp seen on a larger scale in the HI distribution (Cohen \& Davies 1976).

It is possible that the $\mathrm{OH}$ survey has not traced the full $\mathrm{z}$-extent of the 


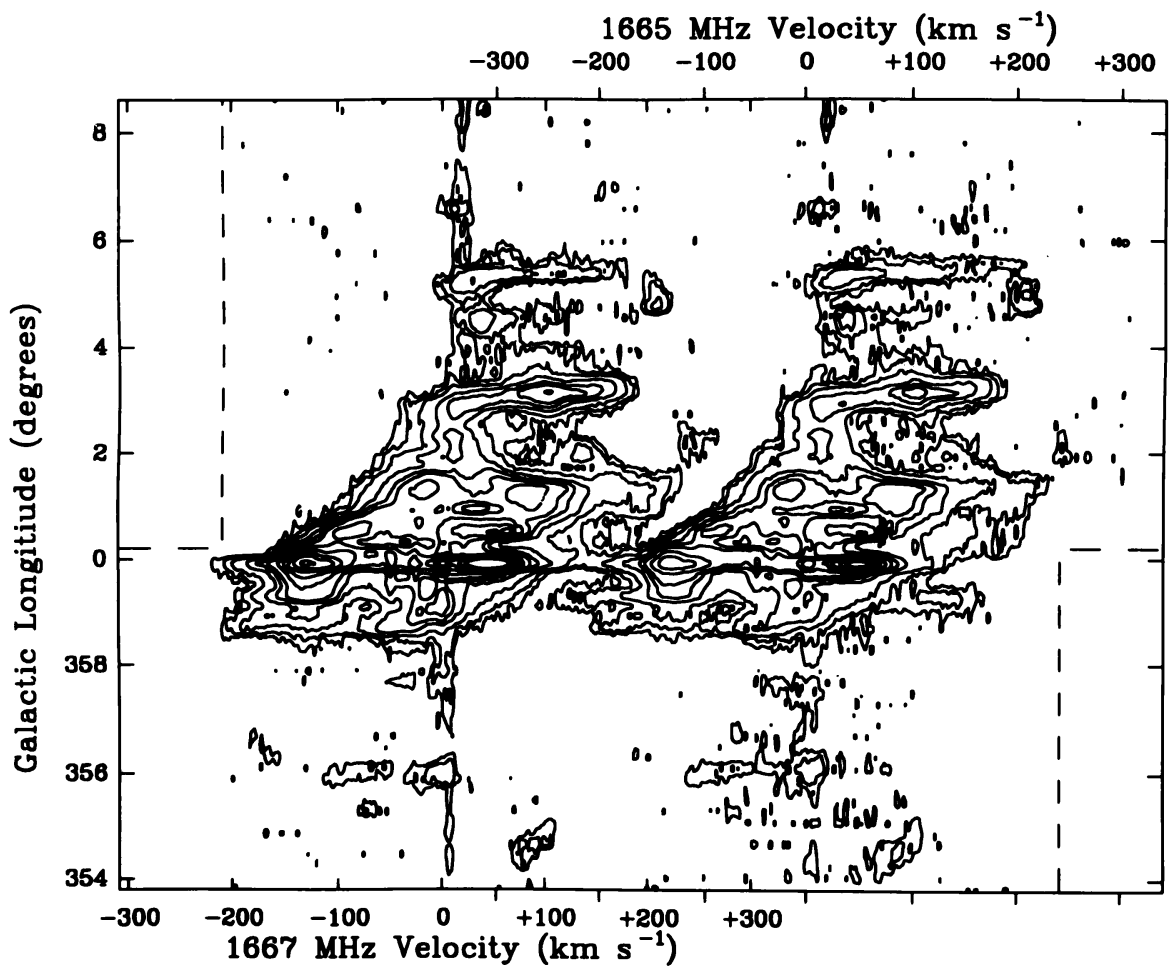

Figure 2. Longitude-velocity map of $\mathrm{OH}$ absorption integrated over galactic latitude. Contour levels are in units of antenna temperature and are set at $-0.75,-1.5,-3,-6,-9$, $-12,-18,-24,-30,-36$ and $-42 \mathrm{~K}$. The resolution is $3.1 \mathrm{~km} \mathrm{~s}^{-1} \times 12^{\prime}$.

molecular distribution. There are a few positions where $\mathrm{OH}$ absorption was detected at the outermost latitudes of the grid, for example at the lower extremities of Spurs IV and V. Furthermore the detection of $\mathrm{OH}$ absorption relies on a continuum background. In the present survey $\mathrm{OH}$ absorption was detected only at positions where the radio continuum was at least $5 \mathrm{~K}$ of antenna temperature (corresponding to $7.5 \mathrm{~K}$ of brightness temperature). Therefore it is possible that molecular gas could have been present far from the galactic plane in regions of low radio continuum emission, and not have been detected in the $\mathrm{OH}$ survey. The $\mathrm{CO}$ emission line observations by Dame et al. (1987) show that there is no large concentration of molecular gas far from the plane $\left(|b|<8^{\circ}\right)$, but those observations would not have been sensitive to narrow filamentary structures such as the molecular spurs 
reported here.

The $\mathrm{OH}$ survey has revealed three new phenomena. Perhaps the most striking are the broadline clump features, of which there are at least 7 . These are concentrations of molecular gas with angular sizes of typically 0.6 and velocity extents of typically $200 \mathrm{~km} \mathrm{~s}^{-1}$. The clumps have positive gradients of velocity with longitude. The clumps are easily recognized in the longitude-velocity diagram Figure 2. The clump parameters are listed in Table 1.

TABLE 1. Properties of broadline clumps

\begin{tabular}{llllll}
\hline Feature & $\begin{array}{l}l \text { range } \\
\mathrm{deg}\end{array}$ & $\begin{array}{l}b \text { range } \\
\mathrm{deg}\end{array}$ & $\begin{array}{l}V \text { range } \\
\mathrm{km} \mathrm{s}^{-1}\end{array}$ & $\begin{array}{l}\mathrm{N}_{O H} / \mathrm{T}_{e x} \\
\times 10^{14} \mathrm{~cm}^{-2} \mathrm{~K}^{-1}\end{array}$ & $\begin{array}{l}\mathrm{M}_{O H} \\
\mathrm{M}_{\odot}\end{array}$ \\
\hline Clump 1 & $354.4 \rightarrow 355.2$ & $0.0 \rightarrow+0.6$ & $+55 \rightarrow+120$ & $2.6 \pm 0.7$ & $1.0 \pm 0.1$ \\
Clump 2 & $2.8 \rightarrow 3.8$ & $-0.4 \rightarrow+0.8$ & $-45 \rightarrow+245$ & $16.0 \pm 2.3$ & $21.6 \pm 2.4$ \\
Clump 3 & $5.2 \rightarrow 6.0$ & $-0.8 \rightarrow+0.2$ & $-30 \rightarrow+210$ & $6.7 \pm 2.3$ & $6.0 \pm 0.7$ \\
Clump 4 & $4.4 \rightarrow 4.8$ & $-1.2 \rightarrow-0.4$ & $+15 \rightarrow+195$ & $6.7 \pm 2.3$ & $2.4 \pm 0.3$ \\
Clump 5 & $1.0 \rightarrow 2.0$ & $-0.6 \rightarrow+0.4$ & $-45 \rightarrow+220$ & $18.4 \pm 2.3$ & $18.4 \pm 2.1$ \\
Clump 6 & $355.8 \rightarrow 356.4$ & $+0.2 \rightarrow+1.2$ & $-120 \rightarrow+30$ & $3.4 \pm 1.1$ & $2.1 \pm 0.7$ \\
Clump 7 & $355.4 \rightarrow 355.6$ & $+0.4 \rightarrow+0.6$ & $+15 \rightarrow+95$ & $2.4 \pm 0.9$ & $0.30 \pm 0.05$ \\
\hline
\end{tabular}

At positive longitudes the clumps appear to be connected by filaments which have typical widths of $30 \mathrm{~km} \mathrm{~s}^{-1}$, much narrower than those of the clumps. The filament parameters are listed in Table 2. Together the clumps and filaments appear to form an organized structure which accounts for most of the molecular gas at $|l|>2^{\circ}$. The clumps at negative longitudes have lower apparent opacities, and it is possible that filaments connecting them would have been below our detection limit.

The third new phenomenon is the existence of molecular spurs near $l=0^{\circ}$ which point out of the galactic plane and extend to latitudes of at least $\pm 1^{\circ}$. The spurs all appear to be associated with radio continuum features. Spurs I and II follow the galactic centre lobe (Sofue \& Handa 1984), Spur III follows the southern galactic centre lobe (Seiradakis et al. 1989), and Spurs IV and V are associated with the shell source G359.1-0.5 (Uchida et al. 1992). Figure 3 presents a comparison of the molecular spurs with the continuum features. $\mathrm{CO}$ observations of these spurs would be desirable to trace their full extents and to study at higher angular resolution their association with the radio continuum features. 
TABLE 2. Properties of filaments

\begin{tabular}{lllllll}
\hline Feature & $\begin{array}{l}l \text { range } \\
\mathrm{deg}\end{array}$ & $\begin{array}{l}b \text { range } \\
\mathrm{deg}\end{array}$ & $\begin{array}{l}V \text { range } \\
\mathrm{km} \mathrm{s}^{-1}\end{array}$ & $\begin{array}{l}\Delta \mathrm{V}_{1 / 2} \\
\mathrm{~km} \mathrm{~s}^{-1}\end{array}$ & $\begin{array}{l}\mathrm{N}_{O H} / \mathrm{T}_{e x} \\
\mathrm{~cm}^{-2} \mathrm{~K}^{-1}\end{array}$ & $\begin{array}{l}\mathrm{M}_{O H} \\
\mathrm{M}_{\odot}\end{array}$ \\
\hline Filament A & $4.6 \rightarrow 5.0$ & $-0.4 \rightarrow 0.0$ & $+20 \rightarrow+50$ & 30 & $1.5 \times 10^{14}$ & 0.4 \\
Filament B & $3.4 \rightarrow 4.2$ & $-1.0 \rightarrow-0.4$ & $+40 \rightarrow+70$ & 18 & $3.7 \times 10^{14}$ & 1.7 \\
Filament C & $5.0 \rightarrow 5.4$ & $-0.8 \rightarrow-0.6$ & $+170 \rightarrow+220$ & 19 & $1.4 \times 10^{14}$ & 0.3 \\
Filament D & $4.8 \rightarrow 5.4$ & $-1.6 \rightarrow-1.2$ & $+185 \rightarrow+220$ & 20 & $1.7 \times 10^{14}$ & 0.4 \\
Filament E & $1.8 \rightarrow 3.0$ & $-0.2 \rightarrow+0.4$ & $-40 \rightarrow+50$ & 35 & $1.2 \times 10^{14}$ & 9.6 \\
Filament F & $1.8 \rightarrow 2.6$ & $-0.4 \rightarrow-0.2$ & $+115 \rightarrow+160$ & 16 & $3.3 \times 10^{14}$ & 0.9 \\
\hline
\end{tabular}

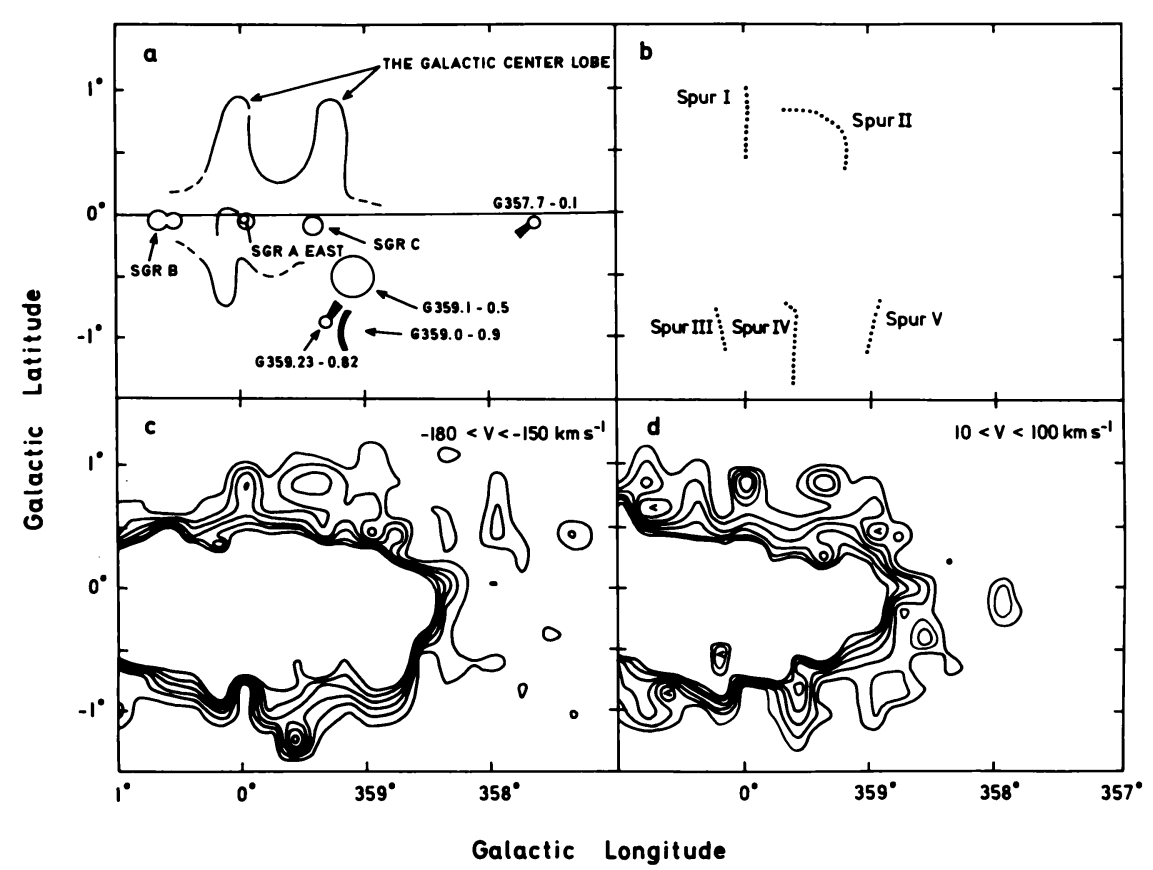

Figure 3. a) a schematic chart of continuum features close to the nucleus (from Uchida et al. 1990), b) a schematic chart of the locations of the molecular spurs, c) a map of the spurs obtained by integrating the $1667 \mathrm{MHz}$ line over $V=-180 \rightarrow-150 \mathrm{~km} \mathrm{~s}^{-1}$. Contours are set at $2,4,6,8,10$ and $12 \mathrm{~K} \mathrm{~km} \mathrm{~s}^{-1}$, d) a map of the spurs obtained by integrating the $1667 \mathrm{MHz}$ line over it $\mathrm{V}=+10 \rightarrow+100 \mathrm{~km} \mathrm{~s}^{-1}$. Contours are set at $2,4,6,8,10$ and $12 \mathrm{~K} \mathrm{~km} \mathrm{~s}^{-1}$.

\section{Discussion of clumps}

The most difficult features to explain are the broadline clumps, which appear to be a new phenomenon confined to the galactic centre. The masses 
of the clumps can be estimated in various ways to be about $10^{7} M_{\odot}$. They usually contain substructure, with length scales of $15 \mathrm{pc}$ and velocity widths of $20 \mathrm{~km} \mathrm{~s}^{-1}$. Recent measurements at Jodrell Bank reveal that the clumps also have a substantial component of neutral atomic hydrogen with a mass of typically $3 \times 10^{5} \mathrm{M}_{\odot}$ (Nixon 1994). The large number of these clumps, and the fact that they show positive gradients of velocity with longitude, put strong constraints on their possible origin. From an observational point of view the clumps have some similarities to the enormous concentrations of molecular gas seen in the nuclei of starburst galaxies and megamaser galaxies. However if the clumps are considered as compact entities then they would have enormous kinetic energies of expansion, typically $10^{54}$ ergs. On the other hand if they were to be incorporated into a bar-type model for the gas kinematics of the central region then they would translate from wide lines in $l-V$ space to long dust lanes in the central region, all pointing exactly towards the Sun. Neither of these models seems entirely satisfactory at the present time.

\section{References}

Bally, J., Stark, A.A., Wilson, R.W., Henkel, C., 1987, ApJS 65, 13

Bally, J., Stark, A.A., Wilson, R.W., Henkel, C., 1988, ApJ 324, 223

Bania, T.M. 1977, ApJ 216, 381

Boyce, P.J., Cohen, R.J. 1994 A\&AS 107, 563

Cohen, R.J., Davies, R.D. 1976, MNRAS 175, 1

Cohen, R.J., Few, R.W. 1976, MNRAS 176, 495

Cohen, R.J., Few, R.W. 1981, MNRAS 194, 711

Dame, T.M., Ungerechts, H., Cohen, R.S., et. al. 1987, ApJ 322, 706

Nixon, C.A. 1994, MSc thesis, University of Manchester

Robinson, B.J., McGee, R.X. 1970, Aust.J.Phys 23, 405

Seiradakis, J.H., Reich, W., Sofue, Y. 1989, ed. M. Morris, The Centre of our Galaxy, I.A.U. Symp. No.136 (Kluwer Academic Publishers, Dordrecht) 237

Sofue, Y., Handa, T. 1984, Nature 310, 568

Uchida, K.I., Morris, M., Bally, J., Pound, M., Yusef-Zadeh, F. 1992, ApJ 398, 128

Whiteoak, J.B., Gardener, F.F. 1979, MNRAS 188, 445 